
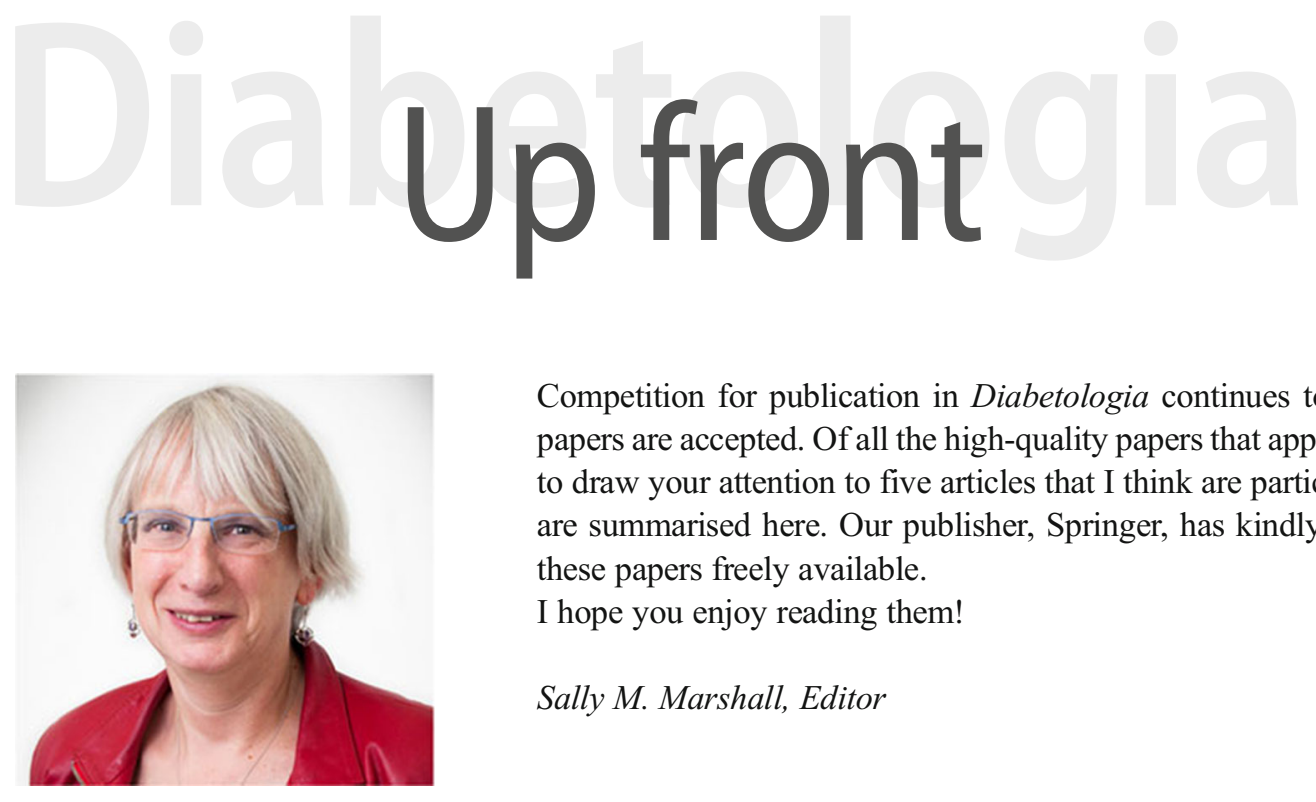

Competition for publication in Diabetologia continues to grow, and less than $20 \%$ of papers are accepted. Of all the high-quality papers that appear in this month's issue I want to draw your attention to five articles that I think are particularly interesting. The articles are summarised here. Our publisher, Springer, has kindly made the full text of each of these papers freely available.

I hope you enjoy reading them!

Sally M. Marshall, Editor

\section{Neuronal control of peripheral nutrient partitioning}

Romane Manceau, Danie Majeur, Thierry Alquier

In addition to food intake and body weight regulation, it has become clear that specific brain regions and neurocircuits orchestrate the metabolic fate of nutrients in peripheral tissues in rodents and humans. Increasing evidence suggests that alterations in these processes can contribute to the development of obesity and diabetes. In this issue, Manceau et al (https://doi.org/10.1007/s00125-020-05104-9) review historical evidence and recent studies demonstrating that specific neuronal populations of the hypothalamus regulate the utilisation, storage and conversion of nutrients (referred to as nutrient partitioning) in peripheral tissues via the autonomic nervous system. In addition, this review summarises the molecular and biochemical mechanisms involved in regulation of peripheral nutrient partitioning by hypothalamic cells. Finally, the authors discuss the contribution of impaired neuronal control of nutrient partitioning to metabolic pathologies in humans.

(b) The figures from this review are available as a downloadable slideset

\section{The changing face of paediatric diabetes}

Amy S. Shah, Kristen J. Nadeau

Paediatric diabetes is on the rise. In the last decade there has been extensive research dedicated to understanding its pathophysiology and its associated comorbidities and complications. In this issue, Shah and Nadeau (https://doi.org/10. 1007/s00125-019-05075-6) summarise the current state of knowledge of type 1 and type 2 diabetes in children and adolescents. They review the current epidemiology and address the importance of differentiating between the two main types of diabetes, especially in the setting of obesity. The authors go on to discuss the observed comorbidities and complications in type 1 and type 2 diabetes in youth, pointing out that abnormalities in heart physiology, blood dynamics and renal hyperfiltration are more common in individuals with either type of diabetes than in peers without diabetes. Finally, currently available treatments and novel treatments on the horizon are discussed.

(b) The figure from this review is available as a downloadable slide.

Persistent poor glycaemic control in individuals with type 2 diabetes in developing countries: 12 years of real-world evidence of the International Diabetes Management Practices Study (IDMPS)

Pablo Aschner, Juan J. Gagliardino, Hasan Ilkova, Fernando Lavalle, Ambady Ramachandran, Jean Claude Mbanya, Marina Shestakova, Jean-Marc Chantelot, Juliana C. N. Chan

Management of type 2 diabetes has advanced considerably over the past decade, but there are limited data from developing countries. The International Diabetes Management 
Practices Study (IDMPS) is an ongoing, international, observational study that documents current practices in diabetes management in developing countries. In this issue, Aschner et al (https://doi.org/10.1007/s00125-019-05078-3) report that data from across seven cross-sectional 'waves', from 2005 to 2017 (including $>65,000$ people with type 2 diabetes), indicate that $\mathrm{HbA}_{1 \mathrm{c}}$ target attainment in these countries remains poor and has, in fact, decreased significantly over time; this is despite increased use of insulin and a higher proportion of patients undergoing frequent $\mathrm{HbA}_{1 \mathrm{c}}$ measurements. While receipt of diabetes education increased over time, this was predominantly provided only by physicians. In summary, the authors state that, while some encouraging trends in care improvements were observed, system changes are needed to improve access to structured education, self-monitoring tools and appropriate medications. They propose that such changes will support self-management and enable care providers to intensify treatment as recommended in guidelines.

\section{Obesity-induced reduced expression of the IncRNA ROIT impairs insulin transcription by downregulation of $N k x 6.1$ methylation}

Fang Fang Zhang, Yu Hong Liu, Dan Wei Wang, Ting Sheng Liu, Yue Yang, Jia Min Guo, Yi Pan, Yan Feng Zhang, Hong Du, Ling Li, Liang Jin

Abnormal expression of long non-coding RNAs (lncRNAs) has been associated with a variety of human diseases, including diabetes. However, it is unclear whether islet lncRNAs are involved in obesity-mediated beta cell dysfunction, which is associated with diabetes. In this issue, Zhang et al (https://doi. org/10.1007/s00125-020-05090-y) report that obesity reduces the expression of the lncRNA 1810019D21Rik (referred to as regulator of insulin transcription [ROIT]), as assessed by RNA sequencing analysis. They also show that over- expression of ROIT lncRNA leads to improved glucose homeostasis and insulin transcription. The authors suggest that this may be because ROIT IncRNA can downregulate the methylation of the $N k x 6.1$ promoter by binding to DNA methyltransferase $3 \mathrm{a}$ and causing its degradation. The authors conclude that these findings indicate that at least some of the harmful effects of obesity on beta cell function may be mediated by obesity-induced alterations in the level of the ROIT lncRNA.

An adipocyte-specific defect in oxidative phosphorylation increases systemic energy expenditure and protects against diet-induced obesity in mouse models

Min Jeong Choi, Saet-Byel Jung, Seong Eun Lee, Seul Gi Kang, Ju Hee Lee, Min Jeong Ryu, Hyo Kyun Chung, Joon Young Chang, Yong Kyung Kim, Hyun Jung Hong, Hail Kim, Hyun Jin Kim, Chul-Ho Lee, Adil Mardinoglu, Hyon-Seung Yi, Minho Shong

Adipose tissue mitochondrial oxidative phosphorylation is critical for systemic energy homeostasis, and its dysfunction has been suggested in the aetiology of insulin resistance and diabetes. To date, there is limited experimental evidence on the systemic effects of mitochondrial oxidative phosphorylation on glucose and energy homeostasis. In this issue, Choi et al (https://doi.org/10.1007/s00125-019-05082-7) report that knockout of the gene encoding mitochondrial large ribosomal subunit protein (CRIF1) paradoxically improves systemic energy homeostasis via cell-autonomous activation of the mitochondrial unfolded protein response, and via the noncell-autonomous mitokine actions of growth differentiation factor 15 (GDF15) and fibroblast growth factor 21 (FGF21). The authors state that these findings suggest that mitokines and their receptor modulators may be potential targets for the treatment of obesity and metabolic disease.

All text supplied by the authors. 